\title{
Reducing Weight Undertraining in Structured Discriminative Learning
}

\author{
Charles Sutton, Michael Sindelar, and Andrew McCallum \\ Department of Computer Science \\ University of Massachusetts Amherst \\ Amherst, MA 01003 USA \\ \{casutton, mccallum\}@cs.umass.edu, msindela@student.umass.edu
}

\begin{abstract}
Discriminative probabilistic models are very popular in NLP because of the latitude they afford in designing features. But training involves complex trade-offs among weights, which can be dangerous: a few highlyindicative features can swamp the contribution of many individually weaker features, causing their weights to be undertrained. Such a model is less robust, for the highly-indicative features may be noisy or missing in the test data. To ameliorate this weight undertraining, we introduce several new feature bagging methods, in which separate models are trained on subsets of the original features, and combined using a mixture model or a product of experts. These methods include the logarithmic opinion pools used by Smith et al. (2005). We evaluate feature bagging on linear-chain conditional random fields for two natural-language tasks. On both tasks, the feature-bagged CRF performs better than simply training a single CRF on all the features.
\end{abstract}

\section{Introduction}

Discriminative methods for training probabilistic models have enjoyed wide popularity in natural language processing, such as in part-of-speech tagging (Toutanova et al., 2003), chunking (Sha and Pereira, 2003), namedentity recognition (Florian et al., 2003; Chieu and Ng, 2003), and most recently parsing (Taskar et al., 2004). A discriminative probabilistic model is trained to maximize the conditional probability $p(\mathbf{y} \mid \mathbf{x})$ of output labels $\mathbf{y}$ given input variables $\mathbf{x}$, as opposed to modeling the joint probability $p(\mathbf{y}, \mathbf{x})$, as in generative models such as the Naive Bayes classifier and hidden Markov models. The popularity of discriminative models stems from the great flexibility they allow in defining features: because the distribution over input features $p(\mathbf{x})$ is not modeled, it can contain rich, highly overlapping features without making the model intractable for training and inference.

In NLP, for example, useful features include word bigrams and trigrams, prefixes and suffixes, membership in domain-specific lexicons, and information from semantic databases such as WordNet. It is not uncommon to have hundreds of thousands or even millions of features.

But not all features, even ones that are carefully engineered, improve performance. Adding more features to a model can hurt its accuracy on unseen testing data. One well-known reason for this is overfitting: a model with more features has more capacity to fit chance regularities in the training data. In this paper, however, we focus on another, more subtle effect: adding new features can cause existing ones to be underfit. Training of discriminative models, such as regularized logistic regression, involves complex trade-offs among weights. A few highlyindicative features can swamp the contribution of many individually weaker features, even if the weaker features, taken together, are just as indicative of the output. Such a model is less robust, for the few strong features may be noisy or missing in the test data.

This effect was memorably observed by Dean Pomerleau (1995) when training neural networks to drive vehicles autonomously. Pomerleau reports one example when the system was learning to drive on a dirt road:

The network had no problem learning and then driving autonomously in one direction, but when driving the other way, the network was erratic, swerving from one side of the road to the other. ...It turned out that the network was basing most of its predictions on an easilyidentifiable ditch, which was always on the right in the training set, but was on the left when the vehicle turned around. (Pomerleau, 1995)

The network had features to detect the sides of the road, and these features were active at training and test time, although weakly, because the dirt road was difficult to 
detect. But the ditch was so highly indicative that the network did not learn the dependence between the road edge and the desired steering direction.

A natural way of avoiding undertraining is to train separate models for groups of competing features-in the driving example, one model with the ditch features, and one with the side-of-the-road features-and then average them into a single model. This is same idea behind logarithmic opinion pools, used by Smith, Cohn, and Osborne (2005) to reduce overfitting in CRFs. In this paper, we tailor our ensemble to reduce undertraining rather than overfitting, and we introduce several new combination methods, based on whether the mixture is taken additively or geometrically, and on a per-sequence or pertransition basis. We call this general class of methods feature bagging, by analogy to the well-known bagging algorithm for ensemble learning.

We test these methods on conditional random fields (CRFs) (Lafferty et al., 2001; Sutton and McCallum, 2006), which are discriminatively-trained undirected models. On two natural-language tasks, we show that feature bagging performs significantly better than training a single CRF with all available features.

\section{Conditional Random Fields}

Conditional random fields (CRFs) (Lafferty et al., 2001; Sutton and McCallum, 2006) are undirected graphical models of a conditional distribution. Let $\mathbf{G}$ be an undirected graphical model over random vectors $\mathbf{y}$ and $\mathbf{x}$. As a typical special case, $\mathbf{y}=\left\{y_{t}\right\}$ and $\mathbf{x}=\left\{x_{t}\right\}$ for $t=1, \ldots, T$, so that $\mathbf{y}$ is a labeling of an observed sequence $\mathbf{x}$. For a given collection $C=\left\{\left\{\mathbf{y}_{c}, \mathbf{x}_{c}\right\}\right\}$ of cliques in $\mathbf{G}$, a CRF models the conditional probability of an assignment to labels $\mathbf{y}$ given the observed variables $\mathbf{x}$ as:

$$
p_{\Lambda}(\mathbf{y} \mid \mathbf{x})=\frac{1}{Z(\mathbf{x})} \prod_{c \in C} \Phi\left(\mathbf{y}_{c}, \mathbf{x}_{c}\right),
$$

where $\Phi$ is a potential function and the partition function $Z(\mathbf{x})=\sum_{\mathbf{y}} \prod_{c \in C} \Phi\left(\mathbf{y}_{c}, \mathbf{x}_{c}\right)$ is a normalization factor over all possible label assignments.

We assume the potentials factorize according to a set of features $\left\{f_{k}\right\}$, which are given and fixed, so that

$$
\Phi\left(\mathbf{y}_{c}, \mathbf{x}_{c}\right)=\exp \left(\sum_{k} \lambda_{k} f_{k}\left(\mathbf{y}_{c}, \mathbf{x}_{c}\right)\right)
$$

The model parameters are a set of real weights $\Lambda=\left\{\lambda_{k}\right\}$, one weight for each feature.

Many applications have used the linear-chain $C R F$, in which a first-order Markov assumption is made on the hidden variables. In this case, the cliques of the conditional model are the nodes and edges, so that there are feature functions $f_{k}\left(y_{t-1}, y_{t}, \mathbf{x}, t\right)$ for each label transition. (Here we write the feature functions as potentially

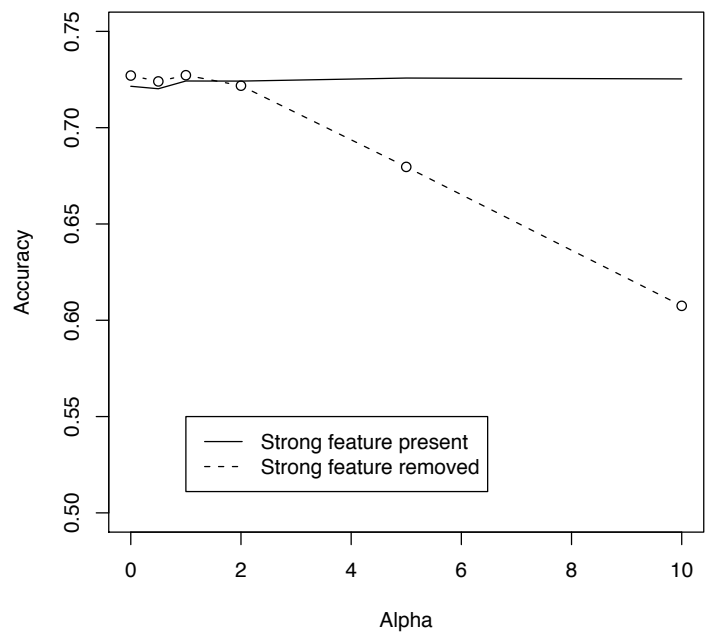

Figure 1: Effect of a single strong feature drowning out weaker features in logistic regression on synthetic data. The $x$-axis indicates the strength of the strong feature. In the top line, the strong feature is present at training and test time. In the bottom line, the strong feature is missing from the training data at test time.

depending on the entire input sequence.) Feature functions can be arbitrary. For example, a feature function $f_{k}\left(y_{t-1}, y_{t}, \mathbf{x}, t\right)$ could be a binary test that has value 1 if and only if $y_{t-1}$ has the label "adjective", $y_{t}$ has the label "proper noun", and $x_{t}$ begins with a capital letter.

Linear-chain CRFs correspond to finite state machines, and can be roughly understood as conditionally-trained hidden Markov models (HMMs). This class of CRFs is also a globally-normalized extension to Maximum Entropy Markov Models (McCallum et al., 2000) that avoids the label bias problem (Lafferty et al., 2001).

Note that the number of state sequences is exponential in the input sequence length $T$. In linear-chain CRFs, the partition function $Z(\mathbf{x})$, the node marginals $p\left(y_{i} \mid \mathbf{x}\right)$, and the Viterbi labeling can be calculated efficiently by variants of the dynamic programming algorithms for HMMs.

\section{Weight Undertraining}

In the section, we give a simple demonstration of weight undertraining. In a discriminative classifier, such as a neural network or logistic regression, a few strong features can drown out the effect of many individually weaker features, even if the weak features are just as indicative put together. To demonstrate this effect, we present an illustrative experiment using logistic regression, because of its strong relation to CRFs. (Linear- 
chain conditional random fields are the generalization of logistic regression to sequence data.)

Consider random variables $x_{1} \ldots x_{n}$, each distributed as independent standard normal variables. The output $y$ is a binary variable whose probability depends on all the $x_{i}$; specifically, we define its distribution as $y \sim$ Bernoulli $\left(\operatorname{logit}\left(\sum_{i} x_{i}\right)\right)$. The correct decision boundary in this synthetic problem is the hyperplane tangent to the weight vector $(1,1, \ldots, 1)$. Thus, if $n$ is large, each $x_{i}$ contributes weakly to the output $y$. Finally, we include a highly indicative feature $x_{S}=\alpha \sum_{i} x_{i}+\mathcal{N}(\mu=$ $\left.0, \sigma^{2}=0.04\right)$. This variable alone is sufficient to determine the distribution of $y$. The variable $\alpha$ is a parameter of the problem that determines how strongly indicative $x_{S}$ is; specifically, when $\alpha=0$, the variable $x_{S}$ is random noise.

We choose this synthetic model by analogy to Pomerleau's observations. The $x_{i}$ correspond to the side of the road in Pomerleau's case-the weak features present at both testing and training - and $x_{S}$ corresponds to the ditch - the strongly indicative feature that is corrupted at test time.

We examine how badly the learned classifier is degraded when $x_{S}$ feature is present at training time but missing at test time. For several values of the weight parameter $\alpha$, we train a regularized logistic regression classifier on 1000 instances with $n=10$ weak variables. In Figure 1, we show how the amount of error caused by ablating $x_{S}$ at test time varies according to the strength of $x_{S}$. Each point in Figure 1 is averaged over 100 randomly-generated data sets. When $x_{S}$ is weakly indicative, it does not affect the predictions of the model at all, and the classifier's performance is the same whether it appears at test time or not. When $x_{S}$ becomes strongly indicative, however, the classifier learns to depend on it, and performs much more poorly when $x_{S}$ is ablated, even though exactly the same information is available in the weak features.

\section{Feature Bagging}

In this section, we describe the feature bagging method. We divide the set of features $F=\left\{f_{k}\right\}$ into a collection of possibly overlapping subsets $\mathcal{F}=\left\{F_{1}, \ldots F_{M}\right\}$, which we call feature bags. We train individual CRFs on each of the feature bags using standard MAP training, yielding individual CRFs $\left\{p_{1}, \ldots p_{M}\right\}$.

We average the individual CRFs into a single combined model. This averaging can be performed in several ways: we can average probabilities of entire sequences, or of individual transitions; and we can average using the arithmetic mean, or the geometric mean. This yields four combination methods:

1. Per-sequence mixture. The distribution over label sequences $\mathbf{y}$ given inputs $\mathbf{x}$ is modeled as a mixture of the individual CRFs. Given nonnegative weights $\left\{\alpha_{1}, \ldots \alpha_{m}\right\}$ that sum to 1 , the combined model is given by

$$
p_{\mathrm{SM}}(\mathbf{y} \mid \mathbf{x})=\sum_{i=1}^{M} \alpha_{i} p_{i}(\mathbf{y} \mid \mathbf{x})
$$

It is easily seen that if the sequence model is defined as in Equation 3, then the pairwise marginals are mixtures as well:

$$
p_{\mathrm{SM}}\left(y_{t}, y_{t-1} \mid \mathbf{x}\right)=\sum_{i=1}^{M} \alpha_{i} p_{i}\left(y_{t}, y_{t-1} \mid \mathbf{x}\right) \text {. }
$$

The probabilities $p_{i}\left(y_{t}, y_{t-1} \mid \mathbf{x}\right)$ are pairwise marginal probabilities in the individual models, which can be efficiently computed by the forward-backward algorithm.

We can perform decoding in the mixture model by maximizing the individual node marginals. That is, to predict $y_{t}$ we compute

$y_{t}^{*}=\arg \max _{y_{t}} p_{\text {SM }}\left(y_{t} \mid \mathbf{x}\right)=\arg \max _{y_{t}} \sum_{i} \alpha_{i} p_{i}\left(y_{t} \mid \mathbf{x}\right)$,

where $p_{i}\left(y_{t} \mid \mathbf{x}\right)$ is computed by first running forward-backward on each of the individual CRFs.

In the results here, however, we compute the maximum probability sequence approximately, as follows. We form a linear-chain distribution $p_{\mathrm{APPX}}(\mathbf{y} \mid \mathbf{x})=\prod_{t} p_{\mathrm{SM}}\left(y_{t} \mid y_{t-1}, \mathbf{x}\right)$, and compute the most probable sequence according to $p_{\mathrm{APPX}}$ by the Viterbi algorithm. This is approximate because $p_{\mathrm{SM}}$ is not a linear-chain distribution in general, even when all the components are. However, the distribution $p_{\mathrm{APPX}}$ does minimize the KL-divergence $D\left(p_{\mathrm{SM}} \| q\right)$ over all linear-chain distributions $q$.

The mixture weights can be selected in a variety of ways, including equal voting, as in traditional bagging, or EM.

2. Per-sequence product of experts. These are the logarithmic opinion pools that have been applied to CRFs by (Smith et al., 2005). The distribution over label sequences $\mathbf{y}$ given inputs $\mathbf{x}$ is modeled as a product of experts (Hinton, 2000). In a product of experts, instead of summing the probabilities from the individual models, we multiply them together. Essentially we take a geometric mean instead of an arithmetic mean. Given nonnegative weights $\left\{\alpha_{1}, \ldots \alpha_{m}\right\}$ that sum to 1 , the product model is

$$
p(\mathbf{y} \mid \mathbf{x}) \propto \prod_{i=1}^{M}\left(p_{i}(\mathbf{y} \mid \mathbf{x})\right)^{\alpha_{i}}
$$


The combined model can also be viewed as a conditional random field whose features are the log probabilities from the original models:

$$
p(\mathbf{y} \mid \mathbf{x}) \propto \exp \left\{\sum_{i=1}^{M} \alpha_{i} \log p_{i}(\mathbf{y} \mid \mathbf{x})\right\}
$$

By substituting in the CRF definition, it can be seen that the model in Equation 7 is simply a single CRF whose parameters are a weighted average of the original parameters. So feature bagging using the product method does not increase the family of models that are considered: standard training of a single CRF on all available features could potentially pick the same parameters as the bagged model.

Nevertheless, in Section 5, we show that this feature bagging method performs better than standard CRF training.

The previous two combination methods combine the individual models by averaging probabilities of entire sequences. Alternatively, in a sequence model we can average probabilities of individual transitions $p_{i}\left(y_{t} \mid y_{t-1}, \mathbf{x}\right)$. Computing these transition probabilities requires performing probabilistic inference in each of the original CRFs, because $p_{i}\left(y_{t} \mid y_{t-1}, \mathbf{x}\right)=$ $\sum_{\mathbf{y} \backslash y_{t}, y_{t+1}} p\left(\mathbf{y} \mid y_{t-1}, \mathbf{x}\right)$.

This yields two other combination methods:

3. Per-transition mixture. The transition probabilities are modeled as

$$
p_{\text {Тм }}\left(y_{t} \mid y_{t-1}, \mathbf{x}\right)=\sum_{i=1}^{M} \alpha_{i} p_{i}\left(y_{t} \mid y_{t-1}, \mathbf{x}\right)
$$

Intuitively, the difference between per-sequence and per-transition mixtures can be understood generatively. In order to generate a label sequence $\mathbf{y}$ given an input $\mathbf{x}$, the per-sequence model selects a mixture component, and then generates $\mathbf{y}$ using only that component. The per-transition model, on the other hand, selects a component, generates $y_{1}$ from that component, selects another component, generates $y_{2}$ from the second component given $y_{1}$, and so on.

4. Per-transition product of experts. Finally, we can combine the transition distributions using a product model

$$
p_{\mathrm{SP}}\left(y_{t} \mid y_{t-1}, \mathbf{x}\right) \propto \prod_{i=1}^{M} p\left(y_{t} \mid y_{t-1}, \mathbf{x}\right)^{\alpha_{i}}
$$

Each transition distribution is thus-similarly to the per-sequence case - an exponential-family distribution whose features are the log transition probabilities from the individual models. Unlike the per-sequence product, there is no weight-averaging trick here, because the probabilities $p\left(y_{t} \mid y_{t-1}, \mathbf{x}\right)$ are marginal probabilities.

Considered as a sequence distribution $p(\mathbf{y} \mid \mathbf{x})$, the per-transition product is a locally-normalized maximum-entropy Markov model (McCallum et al., 2000). It would not be expected to suffer from label bias, however, because each of the features take the future into account; they are marginal probabilities from CRFs.

Of these four combination methods, Method 2, the persequence product of experts, is originally due to Smith et al. (2005). The other three combination methods are as far as we know novel. In the next section, we compare the four combination methods on several sequence labeling tasks. Although for concreteness we describe them in terms of sequence models, they may be generalized to arbitrary graphical structures.

\section{Results}

We evaluate feature bagging on two natural language tasks, named entity recognition and noun-phrase chunking. We use the standard CoNLL 2003 English data set, which is taken from Reuters newswire and consists of a training set of 14987 sentences, a development set of 3466 sentences, and a testing set of 3684 sentences. The named-entity labels in this data set corresponding to people, locations, organizations and other miscellaneous entities. Our second task is noun-phrase chunking. We use the standard CoNLL 2000 data set, which consists of 8936 sentences for training and 2012 sentences for testing, taken from Wall Street Journal articles annotated by the Penn Treebank project. Although the CoNLL 2000 data set is labeled with other chunk types as well, here we use only the NP chunks.

As is standard, we compute precision and recall for both tasks based upon the chunks (or named entities for CoNLL 2003) as

$$
\begin{aligned}
P & =\frac{\# \text { correctly labeled chunks }}{\# \text { labeled chunks }} \\
R & =\frac{\# \text { correctly labeled chunks }}{\# \text { actual chunks }}
\end{aligned}
$$

We report the harmonic mean of precision and recall as $F_{1}=(2 P R) /(P+R)$.

For both tasks, we use per-sequence product-of-experts feature bagging with two feature bags which we manually choose based on prior experience with the data set. For each experiment, we report two baseline CRFs, one trained on union of the two feature sets, and one trained only on the features that were present in both bags, such as lexical identity and regular expressions. In both data 
sets, we trained the individual CRFs with a Gaussian prior on parameters with variance $\sigma^{2}=10$.

For the named entity task, we use two feature bags based upon character ngrams and lexicons. Both bags contain a set of baseline features, such as word identity and regular expressions (Table 4). The ngram CRF includes binary features for character ngrams of length 2 , 3 , and 4 and word prefixes and suffixes of length 2,3 , and 4. The lexicon CRF includes membership features for a variety of lexicons containing people names, places, and company names. The combined model has 2,342,543 features. The mixture weight $\alpha$ is selected using the development set.

For the chunking task, the two feature sets are selected based upon part of speech and lexicons. Again, a set of baseline features are used, similar to the regular expressions and word identity features used on the named entity task (Table 4). The first bag also includes part-of-speech tags generated by the Brill tagger and the conjunctions of those tags used by Sha and Pereira (2003). The second bag uses lexicon membership features for lexicons containing names of people, places, and organizations. In addition, we use part-of-speech lexicons generated from the entire Treebank, such as a list of all words that appear as nouns. These lists are also used by the Brill tagger (Brill, 1994). The combined model uses 536,203 features. The mixture weight $\alpha$ is selected using 2 -fold cross validation. The chosen model had weight 0.55 on the lexicon model, and weight 0.45 on the ngram model.

In both data sets, the bagged model performs better than the single CRF trained with all of the features. For the named entity task, bagging improves performance from $85.45 \%$ to $86.61 \%$, with a substantial error reduction of $8.32 \%$. This is lower than the best reported results for this data set, which is $89.3 \%$ (Ando and Zhang, 2005), using a large amount of unlabeled data. For the chunking task, bagging improved the performance from $94.34 \%$ to $94.77 \%$, with an error reduction of $7.60 \%$. In both data sets, the improvement is statistically significant (McNemar's test; $p<0.01$ ).

On the chunking task, the bagged model also outperforms the models of Kudo and Matsumoto (2001) and Sha and Pereira (2003), and equals the currently-best results of (Ando and Zhang, 2005), who use a large amount of unlabeled data. Although we use lexicons that were not included in the previous models, the additional features actually do not help the original CRF. Only with feature bagging do these lexicons improve performance.

Finally, we compare the four bagging methods of Section 4: pre-transition mixture, pre-transition product of experts, and per-sequence mixture. On the named entity data, all four models perform in a statistical tie, with no statistically significant difference in their performance (Table 1). As we mentioned in the last section, the de-

\begin{tabular}{|l|c|}
\hline Model & F1 \\
\hline Per-sequence Product of Experts & 86.61 \\
\hline Per-transition Product of Experts & 86.58 \\
\hline Per-sequence Mixture & 86.46 \\
\hline Per-transition Mixture & 86.42 \\
\hline
\end{tabular}

Table 1: Comparison of various bagging methods on the CoNLL 2003 Named Entity Task.

\begin{tabular}{|l|c|}
\hline Model & F1 \\
\hline Single CRF(Base Feat.) & 81.52 \\
\hline Single CRF(All Feat.) & 85.45 \\
\hline \hline Combined CRF & 86.61 \\
\hline
\end{tabular}

Table 2: Results for the CoNLL 2003 Named Entity Task. The bagged CRF performs significantly better than a single CRF with all available features (McNemar's test; $p<0.01)$.

coding procedure for the per-sequence mixture is approximate. It is possible that a different decoding procedure, such as maximizing the node marginals, would yield better performance.

\section{Previous Work}

In the machine learning literature, there is much work on ensemble methods such as stacking, boosting, and bagging. Generally, the ensemble of classifiers is generated by training on different subsets of data, rather than different features. However, there is some literature within unstructured classified on combining models trained on feature subsets. Ho (1995) creates an ensemble of decision trees by randomly choosing a feature subset on which to grow each tree using standard decision tree learners. Other work along these lines include that of Bay (1998) using nearest-neighbor classifiers, and more recently Bryll et al (2003). Also, in Breiman's work on random forests (2001), ensembles of random decision trees are constructed by choosing a random feature at each node. This literature mostly has the goal of improving accuracy by reducing the classifier's variance, that is, reducing overfitting.

In contrast, O'Sullivan et al. (2000) specifically focus on increasing robustness by training classifiers to use all of the available features. Their algorithm FeatureBoost is analogous to AdaBoost, except that the meta-learning algorithm maintains weights on features instead of on instances. Feature subsets are automatically sampled based on which features, if corrupted, would most affect the ensemble's prediction. They show that FeatureBoost is more robust than AdaBoost on synthetically corrupted UCI data sets. Their method does not easily extend to sequence models, especially natural-language models with hundreds of thousands of features. 


\begin{tabular}{|l|c|}
\hline Model & F1 \\
\hline \hline Single CRF(Base Feat.) & 89.60 \\
\hline Single CRF(All Feat.) & 94.34 \\
\hline (Sha and Pereira, 2003) & 94.38 \\
\hline (Kudo and Matsumoto, 2001) & 94.39 \\
\hline (Ando and Zhang, 2005) & 94.70 \\
\hline \hline Combined CRF & 94.77 \\
\hline
\end{tabular}

Table 3: Results for the CoNLL 2000 Chunking Task. The bagged CRF performs significantly better than a single CRF (McNemar's test; $p<0.01$ ), and equals the results of (Ando and Zhang, 2005), who use a large amount of unlabeled data.

\begin{tabular}{l}
$w_{t}=w$ \\
$w_{t}$ begins with a capital letter \\
$w_{t}$ contains only capital letters \\
$w_{t}$ is a single capital letter \\
$w_{t}$ contains some capital letters and some lowercase \\
$w_{t}$ contains a numeric character \\
$w_{t}$ contains only numeric characters \\
$w_{t}$ appears to be a number \\
$w_{t}$ is a string of at least two periods \\
$w_{t}$ ends with a period \\
$w_{t}$ contains a dash \\
$w_{t}$ appears to be an acronym \\
$w_{t}$ appears to be an initial \\
$w_{t}$ is a single letter \\
$w_{t}$ contains punctuation \\
$w_{t}$ contains quotation marks \\
$P_{t}=P$ \\
All features for time $t+\delta$ for all $\delta \in[-2,2]$ \\
\hline
\end{tabular}

Table 4: Baseline features used in all bags. In the above $w_{t}$ is the word at position $t, P_{t}$ is the POS tag at position $t, w$ ranges over all words in the training data, and $P$ ranges over all chunk tags supplied in the training data. The "appears to be" features are based on hand-designed regular expressions.

There is less work on ensembles of sequence models, as opposed to unstructured classifiers. One example is Altun, Hofmann, and Johnson (2003), who describe a boosting algorithm for sequence models, but they boost instances, not features. In fact, the main advantage of their technique is increased model sparseness, whereas in this work we aim to fully use more features to increase accuracy and robustness.

Most closely related to the present work is that on $\log$ arithmic opinion pools for CRFs (Smith et al., 2005), which we have called per-sequence mixture of experts in this paper. The previous work focuses on reducing overfitting, combining a model of many features with several simpler models. In contrast, here we apply feature bagging to reduce feature undertraining, combining several models with complementary feature sets. Our current positive results are probably not due to reduction in over- fitting, for as we have observed, all the models we test, including the bagged one, have $99.9 \% \mathrm{~F} 1$ on the training set. Now, feature undertraining can be viewed as a type of overfitting, because it arises when a set of features is more indicative in the training set than the testing set. Understanding this particular type of overfitting is useful, because it motivates the choice of feature bags that we explore in this work. Indeed, one contribution of the present work is demonstrating how a careful choice of feature bags can yield state-of-the-art performance.

Concurrently and independently, Smith and Osborne (2006) present similar experiments on the CoNLL-2003 data set, examining a per-sequence mixture of experts (that is, a logarithmic opinion pool), in which the lexicon features are trained separately. Their work presents more detailed error analysis than we do here, while we present results both on other combination methods and on NP chunking.

\section{Conclusion}

Discriminatively-trained probabilistic models have had much success in applications because of their flexibility in defining features, but sometimes even highlyindicative features can fail to increase performance. We have shown that this can be due to feature undertraining, where highly-indicative features prevent training of many weaker features. One solution to this is feature bagging: repeatedly selecting feature subsets, training separate models on each subset, and averaging the individual models.

On large, real-world natural-language processing tasks, feature bagging significantly improves performance, even with only two feature subsets. In this work, we choose the subsets based on our intuition of which features are complementary for this task, but automatically determining the feature subsets is an interesting area for future work.

\section{Acknowledgments}

We thank Andrew Ng, Hanna Wallach, Jerod Weinman, and Max Welling for helpful conversations. This work was supported in part by the Center for Intelligent Information Retrieval, in part by the Defense Advanced Research Projects Agency (DARPA), in part by The Central Intelligence Agency, the National Security Agency and National Science Foundation under NSF grant \#IIS0326249, and in part by The Central Intelligence Agency, the National Security Agency and National Science Foundation under NSF grant \#IIS-0427594. Any opinions, findings and conclusions or recommendations expressed in this material are the author(s) and do not necessarily reflect those of the sponsor. 


\section{References}

Yasemin Altun, Thomas Hofmann, and Mark Johnson. 2003. Discriminative learning for label sequences via boosting. In Advances in Neural Information Processing Systems (NIPS*15).

Rie Ando and Tong Zhang. 2005. A high-performance semi-supervised learning method for text chunking. In Proceedings of the 43rd Annual Meeting of the Association for Computational Linguistics (ACL'05), pages 1-9, Ann Arbor, Michigan, June. Association for Computational Linguistics.

Stephen D. Bay. 1998. Combining nearest neighbor classifiers through multiple feature subsets. In ICML '98: Proceedings of the Fifteenth International Conference on Machine Learning, pages 37-45. Morgan Kaufmann Publishers Inc.

Leo Breiman. 2001. Random forests. Machine Learning, 45(1):5-32, October.

Eric Brill. 1994. Some advances in transformation-based part of speech tagging. In AAAI '94: Proceedings of the twelfth national conference on Artificial intelligence (vol. 1), pages 722-727. American Association for Artificial Intelligence.

Robert Bryll, Ricardo Gutierrez-Osuna, and Francis Quek. 2003. Attribute bagging: improving accuracy of classifier ensembles by using random feature subsets. Pattern Recognition, 36:1291-1302.

Hai Leong Chieu and Hwee Tou Ng. 2003. Named entity recognition with a maximum entropy approach. In Walter Daelemans and Miles Osborne, editors, Proceedings of CoNLL-2003, pages 160-163. Edmonton, Canada.

Radu Florian, Abe Ittycheriah, Hongyan Jing, and Tong Zhang. 2003. Named entity recognition through classifier combination. In Proceedings of CoNLL-2003.

G.E. Hinton. 2000. Training products of experts by minimizing contrastive divergence. Technical Report 2000004, Gatsby Computational Neuroscience Unit.

T. K. Ho. 1995. Random decision forests. In Proc. of the 3rd Int'l Conference on Document Analysis and Recognition, pages 278-282, Montreal, Canada, August.

T. Kudo and Y. Matsumoto. 2001. Chunking with support vector machines. In Proceedings of NAACL-2001.

J. Lafferty, A. McCallum, and F. Pereira. 2001. Conditional random fields: Probabilistic models for segmenting and labeling sequence data. Proc. 18th International Conf. on Machine Learning.
Andrew McCallum, Dayne Freitag, and Fernando Pereira. 2000. Maximum entropy Markov models for information extraction and segmentation. In Proc. 17th International Conf. on Machine Learning, pages 591-598. Morgan Kaufmann, San Francisco, CA.

Joseph O'Sullivan, John Langford, Rich Caruana, and Avrim Blum. 2000. Featureboost: A meta learning algorithm that improves model robustness. In International Conference on Machine Learning.

Dean Pomerleau. 1995. Neural network vision for robot driving. In M. Arbib, editor, The Handbook of Brain Theory and Neural Networks.

Fei Sha and Fernando Pereira. 2003. Shallow parsing with conditional random fields. In Proceedings of HLT-NAACL 2003. Association for Computational Linguistics.

Andrew Smith and Miles Osborne. 2006. Using gazetteers in discriminative information extraction. In CoNLL-X, Tenth Conference on Computational Natural Language Learning.

Andrew Smith, Trevor Cohn, and Miles Osborne. 2005. Logarithmic opinion pools for conditional random fields. In Proceedings of the 43rd Annual Meeting of the Association for Computational Linguistics (ACL'05), pages 18-25, Ann Arbor, Michigan, June. Association for Computational Linguistics.

Charles Sutton and Andrew McCallum. 2006. An introduction to conditional random fields for relational learning. In Lise Getoor and Ben Taskar, editors, Introduction to Statistical Relational Learning. MIT Press. To appear.

Ben Taskar, Dan Klein, Michael Collins, Daphne Koller, and Chris Manning. 2004. Max-margin parsing. In Empirical Methods in Natural Language Processing (EMNLP04).

Kristina Toutanova, Dan Klein, Christopher D. Manning, and Yoram Singer. 2003. Feature-rich part-of-speech tagging with a cyclic dependency network. In HLTNAACL 2003. 